\title{
Graph Operations and Neighbor Rupture Degree
}

\author{
Saadet Kandİlci,, ${ }^{1}$ Goksen Bacak-Turan, ${ }^{2}$ and Refet Polat ${ }^{1}$ \\ ${ }^{1}$ Department of Mathematics, Yasar University, 35100 Izmir, Turkey \\ ${ }^{2}$ Department of Mathematics, Celal Bayar University, 45140 Manisa, Turkey \\ Correspondence should be addressed to Goksen Bacak-Turan; goksen.turan@cbu.edu.tr
}

Received 25 March 2013; Revised 13 June 2013; Accepted 14 June 2013

Academic Editor: Frank Werner

Copyright (C) 2013 Saadet Kandilci et al. This is an open access article distributed under the Creative Commons Attribution License, which permits unrestricted use, distribution, and reproduction in any medium, provided the original work is properly cited.

\begin{abstract}
In a communication network, the vulnerability parameters measure the resistance of the network to disruption of operation after the failure of certain stations or communication links. A vertex subversion strategy of a graph $G$, say $S$, is a set of vertices in $G$ whose closed neighborhood is removed from $G$. The survival subgraph is denoted by $G / S$. The neighbor rupture $\operatorname{degree~of~} G, \operatorname{Nr}(G)$, is defined to be $\operatorname{Nr}(G)=\max \{w(G / S)-|S|-c(G / S): S \subset V(G), w(G / S) \geq 1\}$, where $S$ is any vertex subversion strategy of $G, w(G / S)$ is the number of connected components in $G / S$ and $c(G / S)$ is the maximum order of the components of $G / S$ (G. Bacak Turan, 2010). In this paper we give some results for the neighbor rupture degree of the graphs obtained by some graph operations.
\end{abstract}

\section{Introduction}

A network can be broke down completely or partially with unexpected reasons. If the data is not transmitted to the desired location that means there is a problem on the system. This problem can block a treaty of billions of euros or make a big problem for human's life. In these days the reliability and the vulnerability of networks are so important. For that reason graphs are taken as a model in the research area of reliability and vulnerability of the networks. Each network center is taken as a vertex and the connections of these vertices are edges of a graph.

A few questions can be asked at this point How can the reliability and the vulnerability of a network be determined? What are the factors of the reliability and the vulnerability? For example, what can be done if there is a problem on the way you are using every day to work? We have two choices; we may give up going to work although we have the risk of dismissal or we can look for another way to work. The question "if there is another way to reach work" may come to our minds. In other words "Has the link connection between home and work completely broken down?". To answer this question, we must know the dimensions of the problem between home and work. The vulnerability of the graph which represents the way between home and work should be searched. In graph theory some vulnerability parameters are defined to measure the vulnerability value of graphs such as connectivity [1], integrity [2], neighbor integrity [3], rupture degree [4], and neighbor rupture degree [5].

Terminology and notation not defined in this paper can be found in [5]. Let $G$ be a simple graph and let $u$ be any vertex of $G$. The set $N(u)=\{v \in V(G) \mid v \neq u ; v$ and $u$ are adjacent $\}$ is the open neighborhood of $u$, and $N[u]=\{u\} \cup N(u)$ is the closed neighborhood of $u$. A vertex $u$ in $G$ is said to be subverted if the closed neighborhood of $u$ is removed from $G$. A set of vertices $S=\left\{u_{1}, u_{2}, \ldots, u_{m}\right\}$ is called a vertex subversion strategy of $G$ if each of the vertices in $S$ has been subverted from $G$. If $S$ has been subverted from the graph $G$, then the remaining graph is called survival graph, denoted by $G / S$.

\section{Basic Results}

In this paper the new vulnerability parameter neighbor rupture degree was studied. The concept of neighbor rupture degree was introduced by Bacak-Turan and Kirlangic in 2011 [5]. The definition of neighbor rupture degree and some results are given below. 
Definition 1 (see [6]). The neighbor rupture degree of a noncomplete connected graph $G$ is defined to be

$\operatorname{Nr}(G)$

$$
=\max \left\{w\left(\frac{G}{S}\right)-|S|-c\left(\frac{G}{S}\right): S \subset V(G), w\left(\frac{G}{S}\right) \geq 1\right\},
$$

where $S$ is any vertex subversion strategy of $G, w(G / S)$ is the number of connected components in $G / S$, and $c(G / S)$ is the maximum order of the components of $G / S$.

In particular, the neighbor rupture degree of a complete graph $K_{n}$ is defined to be $\operatorname{Nr}\left(K_{n}\right)=1-n$. A set $S \subset V(G)$ is said to be Nr-set of $G$ if

$$
\operatorname{Nr}(G)=w\left(\frac{G}{S}\right)-|S|-c\left(\frac{G}{S}\right) .
$$

Some known results are listed below.

Theorem 2 (see [6]). (a) Let $P_{n}$ be a path graph with $n$ vertices and $n \geq 2$,

$$
\operatorname{Nr}\left(P_{n}\right)= \begin{cases}0, & n \equiv 1(\bmod 4) \\ -1, & n \equiv 0,2,3(\bmod 4) .\end{cases}
$$

(b) Let $C_{n}$ be a cycle graph with $n$ vertices and $n \geq 3$,

$$
\operatorname{Nr}\left(C_{n}\right)= \begin{cases}-1, & n \equiv 0(\bmod 4) \\ -2, & n \equiv 1,2,3(\bmod 4) .\end{cases}
$$

(c) Let $K_{n_{1}, n_{2}, n_{3}, \ldots, n_{k}}$ be a $k$-partite graph

$$
\operatorname{Nr}\left(K_{n_{1}, n_{2}, \ldots, n_{k}}\right)=\max \left\{n_{1}, n_{2}, n_{3}, \ldots, n_{k}\right\}-3 .
$$

(d) Let $W_{n}$ be a wheel graph with $n$ vertices and $n \geq 5$,

$$
\operatorname{Nr}\left(W_{n}\right)= \begin{cases}-1, & n \equiv 1(\bmod 4) \\ -2, & n \equiv 0,2,3(\bmod 4) .\end{cases}
$$

\section{Graph Operations and Neighbor Rupture Degree}

In this section some graph operations are operated on graphs and their neighbor rupture degrees are evaluated.

Definition 3 (see [7]). The union graph $G=G_{1} \cup G_{2} \cup \cdots \cup G_{n}$ has vertex set $V(G)=V\left(G_{1}\right) \cup V\left(G_{2}\right) \cup \cdots \cup V\left(G_{n}\right)$ and edge set $E(G)=E\left(G_{1}\right) \cup E\left(G_{2}\right) \cup \cdots \cup E\left(G_{n}\right)$. If a graph $G$ consists of $k(k \geq 2)$ disjoint copies of a graph $H$, then we write $G=k H$.

Theorem 4. Let $G_{1}, G_{2}, G_{3}, \ldots, G_{n}$ be connected graphs. Then

$$
\begin{aligned}
& \operatorname{Nr}\left(G_{1} \cup G_{2} \cup \cdots \cup G_{n}\right) \\
& \quad \geq \operatorname{Nr}\left(G_{1}\right)+\operatorname{Nr}\left(G_{2}\right)+\cdots+\operatorname{Nr}\left(G_{n}\right) .
\end{aligned}
$$

Proof. Let $G=G_{1} \cup G_{2} \cup \cdots \cup G_{n}$ be the union of $G_{1}, G_{2}, \ldots, G_{n}$. Let $S_{1}, S_{2}, \ldots, S_{n}$ be Nr-sets of $G_{1}, G_{2}, \ldots, G_{n}$ respectively, and let $S=S_{1} \cup S_{2} \cup \cdots \cup S_{n}$ be a subversion strategy of $G$. Then we obtain

$$
\begin{aligned}
\operatorname{Nr}(G) \geq & w\left(\frac{G}{\left(S_{1} \cup S_{2} \cup \cdots \cup S_{n}\right)}\right)-\left|S_{1} \cup S_{2} \cup \cdots \cup S_{n}\right| \\
& -c\left(\frac{G}{\left(S_{1} \cup S_{2} \cup \cdots \cup S_{n}\right)}\right) \\
= & w\left(\frac{G_{1}}{S_{1}}\right)+w\left(\frac{G_{2}}{S_{2}}\right)+\cdots+w\left(\frac{G_{n}}{S_{n}}\right)-\left|S_{1}\right|-\left|S_{2}\right| \\
& -\cdots\left|S_{n}\right|-\max \left\{c\left(\frac{G_{1}}{S_{1}}\right), c\left(\frac{G_{2}}{S_{2}}\right), \ldots, c\left(\frac{G_{n}}{S_{n}}\right)\right\} \\
\geq & w\left(\frac{G_{1}}{S_{1}}\right)+w\left(\frac{G_{2}}{S_{2}}\right)+\cdots+w\left(\frac{G_{n}}{S_{n}}\right)-\left|S_{1}\right|-\left|S_{2}\right| \\
& -\cdots\left|S_{n}\right|-c\left(\frac{G_{1}}{S_{1}}\right)-c\left(\frac{G_{2}}{S_{2}}\right)-\cdots-c\left(\frac{G_{n}}{S_{n}}\right) \\
= & \operatorname{Nr}\left(G_{1}\right)+\operatorname{Nr}\left(G_{2}\right)+\operatorname{Nr}\left(G_{3}\right)+\cdots+\operatorname{Nr}\left(G_{n}\right) .
\end{aligned}
$$

Thus we have $\operatorname{Nr}\left(G_{1} \cup G_{2} \cup \cdots \cup G_{n}\right) \geq \operatorname{Nr}\left(G_{1}\right)+\operatorname{Nr}\left(G_{2}\right)+$ $\cdots+\operatorname{Nr}\left(G_{n}\right)$.

Theorem 5. Let $K_{n_{1}}, K_{n_{2}}, \ldots, K_{n_{m}}$ be complete graphs with $n_{1} \leq n_{2} \leq n_{m}$ where $n_{i+1}-n_{i} \geq 2 ; \forall i \in Z^{+}$. Then

$$
\operatorname{Nr}\left(K_{n_{1}} \cup K_{n_{2}} \cup \cdots \cup K_{n_{m}}\right)=2-m-n_{1} .
$$

Proof. Let $S$ be a subversion strategy of $K_{n_{1}} \cup K_{n_{2}} \cup \cdots \cup K_{n_{m}}$. Since these are complete graphs, it is obvious that $S$ contains at most one vertex from each $K_{n_{i}}$.

If $|S|=k$, then $w\left(\left(K_{n_{1}} \cup K_{n_{2}} \cup \cdots \cup K_{n_{m}}\right) / S\right)=m-k$ and $c\left(\left(K_{n_{1}} \cup K_{n_{2}} \cup \cdots \cup K_{n_{m}}\right) / S\right) \geq n_{m-k}$. Thus we have $w\left(\left(K_{n_{1}} \cup K_{n_{2}} \cup\right.\right.$ $\left.\left.K_{n_{3}} \cup \cdots \cup K_{n_{m}}\right) / S\right)-|S|-c\left(\left(K_{n_{1}} \cup K_{n_{2}} \cup K_{n_{3}} \cup \cdots \cup K_{n_{m}}\right) / S\right) \leq$ $m-2 k-n_{m-k} \leq m-2 k-n_{1}-2(m-k-1)$ since

$$
\begin{gathered}
n_{i+1}-n_{i} \geq 2, \\
n_{m-k} \geq n_{1}+2(m-k-1) \\
=2-m-n_{1} \Longrightarrow \mathrm{Nr} \leq 2-m-n_{1} .
\end{gathered}
$$

There exist $S^{*}$ such that $\left|S^{*}\right|=m-1, w\left(\left(K_{n_{1}} \cup K_{n_{2}} \cup \cdots \cup\right.\right.$ $\left.\left.K_{n_{m}}\right) / S^{*}\right)=1$ and $c\left(\left(K_{n_{1}} \cup K_{n_{2}} \cup \cdots \cup K_{n_{m}}\right) / S^{*}\right)=n_{1}$. Then we have

$$
\begin{aligned}
& w\left(\frac{\left(K_{n_{1}} \cup K_{n_{2}} \cup \cdots \cup K_{n_{m}}\right)}{S^{*}}\right)-\left|S^{*}\right| \\
& \quad-c\left(\frac{\left(K_{n_{1}} \cup K_{n_{2}} \cup \cdots \cup K_{n_{m}}\right)}{S^{*}}\right) \\
& \quad=2-m-n_{1} \\
& \Longrightarrow \mathrm{Nr} \geq 2-m-n_{1} .
\end{aligned}
$$

From (10) and (11) we obtain $\operatorname{Nr}\left(K_{n_{1}} \cup K_{n_{2}} \cup \cdots \cup K_{n_{m}}\right)=$ $2-m-n_{1}$. 
The following theorem's proof is very similar to that of Theorem 5.

Theorem 6. Let $K_{n_{1}}, K_{n_{2}}, K_{n_{3}}, \ldots, K_{n_{m}}$ be complete graphs with $n_{1} \leq n_{2} \leq n_{3} \cdots \leq n_{m}$ where $n_{i+1}-n_{i} \leq 2$; for all $i \in Z^{+}$. Then

$$
\operatorname{Nr}\left(K_{n_{1}} \cup K_{n_{2}} \cup K_{n_{3}} \cup \cdots \cup K_{n_{m}}\right)=m-n_{m} .
$$

Corollary 7. Let

$$
\operatorname{Nr}\left(K_{n} \cup K_{m}\right)= \begin{cases}2-n, & 2>n-m, \\ -m, & \text { otherwise. }\end{cases}
$$

Definition 8 (see [7]). The join graph $G=G_{1}+G_{2}$ has vertex set $V(G)=V\left(G_{1}\right) \cup V\left(G_{2}\right)$ and edge set $E(G)=E\left(G_{1}\right) \cup$ $E\left(G_{2}\right) \cup\left\{u v \mid u \in V\left(G_{1}\right)\right.$ and $\left.v \in V\left(G_{2}\right)\right\}$.

In this part, neighbor rupture degree of join of some graphs is given.

Theorem 9. Let $G_{1}$ and $G_{2}$ be two connected graphs. Then

$$
\operatorname{Nr}\left(G_{1}+G_{2}\right)=\max \left\{\operatorname{Nr}\left(G_{1}\right), \operatorname{Nr}\left(G_{2}\right)\right\} \text {. }
$$

Proof. Let $S$ be a subversion strategy of $G_{1}+G_{2}$. There are three cases according to the elements of $S$.

Case 1. Let $S=S_{1} \subset V\left(G_{1}\right)$ be the Nr-set of $G_{1}$ such that $w\left(G_{1} / S_{1}\right)-\left|S_{1}\right|-c\left(G_{1} / S_{1}\right)=\operatorname{Nr}\left(G_{1}\right)$. Since any elements from $G_{1}$ are adjacent to every element of $G_{2}$ in $G_{1}+G_{2}$, we have

$$
\begin{aligned}
& w\left(\frac{G_{1}+G_{2}}{S_{1}}\right)-\left|S_{1}\right|-c\left(\frac{G_{1}+G_{2}}{S_{1}}\right) \\
& \quad=w\left(\frac{G_{1}}{S_{1}}\right)-\left|S_{1}\right|-c\left(\frac{G_{1}}{S_{1}}\right)=\operatorname{Nr}\left(G_{1}\right) .
\end{aligned}
$$

Case 2. Let $S=S_{2} \subset V\left(G_{2}\right)$ be the Nr-set of $G_{2}$ such that $w\left(G_{2} / S_{2}\right)-\left|S_{2}\right|-c\left(G_{2} / S_{2}\right)=\operatorname{Nr}\left(G_{2}\right)$. Since any elements from $G_{2}$ are adjacent to every element of $G_{1}$ in $G_{1}+G_{2}$, we have

$$
\begin{aligned}
& w\left(\frac{G_{1}+G_{2}}{S_{2}}\right)-\left|S_{2}\right|-c\left(\frac{G_{1}+G_{2}}{S_{2}}\right) \\
& \quad=w\left(\frac{G_{2}}{S_{2}}\right)-\left|S_{2}\right|-c\left(\frac{G_{2}}{S_{2}}\right)=\operatorname{Nr}\left(G_{2}\right) .
\end{aligned}
$$

Case 3. Let $S \subset V\left(G_{1}\right) \cup V\left(G_{2}\right)$. Since $S$ contains at least one vertex of $V\left(G_{1}\right)$ which is adjacent to all the vertices of $V\left(G_{2}\right)$ and $S$ contains at least one vertex of $V\left(G_{2}\right)$ which is adjacent to all the vertices of $V\left(G_{1}\right)$ in $G_{1}+G_{2}$, then $\left(G_{1}+G_{2}\right) / S$ is empty. It contradicts to the definition of neighbor rupture degree.

By (15) and (16) $\operatorname{Nr}\left(G_{1}+G_{2}\right)=\max \left\{\operatorname{Nr}\left(G_{1}\right), \operatorname{Nr}\left(G_{2}\right)\right\}$.

For three or more disjoint graphs $G_{1}, G_{2}, G_{3}, \ldots, G_{n}$ sequential join $G_{1}+G_{2}+\cdots+G_{n}$ is the graph $\left(G_{1}+G_{2}\right) \cup$ $\left(G_{2}+G_{3}\right) \cup \cdots \cup\left(G_{n-1}+G_{n}\right)[8]$.

The following theorem's proof is very similar to that of Theorem 9.
Theorem 10. Let $G_{1}, G_{2}$, and $G_{3}$ be connected graphs. Then the neighbor rupture degree of sequential join of $G_{1}, G_{2}$, and $G_{3}$ is

$$
\operatorname{Nr}\left(G_{1}+G_{2}+G_{3}\right)=\max \left\{\operatorname{Nr}\left(G_{1} \cup G_{3}\right), \operatorname{Nr}\left(G_{2}\right)\right\} .
$$

Corollary 11. If $G_{2} \cong K_{n}$, then

$$
\operatorname{Nr}\left(G_{1}+G_{2}+G_{3}\right) \geq \operatorname{Nr}\left(G_{1}\right)+\operatorname{Nr}\left(G_{3}\right) \text {. }
$$

Corollary 12. If $n_{1} \leq n_{2} \leq n_{3}$, then

$$
\operatorname{Nr}\left(K_{n_{1}}+K_{n_{2}}+K_{n_{3}}\right)=-n_{3} .
$$

Definition 13 (see [9]). The complement of a simple graph $G$ is obtained by taking the vertices of $G$ and joining two of them whenever they are not joined in $G$ and denoted by $G^{c}$.

Theorem 14. Let $P_{n}$ be a path graph of order $n$. Then

$$
\operatorname{Nr}\left(P_{n}^{c}\right)=-1 \text {. }
$$

Proof. Let $S$ be a subversion strategy of $P_{n}^{c}$ and let $S=\{u\}$ where $u \in V\left(P_{n}\right)$.

Case 1. If $\operatorname{deg}(u)=1$ in $P_{n}$, then $u$ is adjacent to all vertices in $P_{n}^{c}$ except its neighbor in $P_{n}$. It means $|N[u]|=n-1$ in $P_{n}^{c}$, then we have

$$
w\left(\frac{P_{n}^{c}}{S}\right)-|S|-c\left(\frac{P_{n}^{c}}{S}\right)=1-1-1=-1 .
$$

Case 2. If $\operatorname{deg}(u)=2$ in $P_{n}$, then $u$ is adjacent to all vertices in $P_{n}^{c}$ except its neighbors in $P_{n}$. It means $|N[u]|=n-2$ in $P_{n}^{c}$ where the remaining two vertices are adjacent. Therefore,

$$
w\left(\frac{P_{n}^{c}}{S}\right)-|S|-c\left(\frac{P_{n}^{c}}{S}\right)=1-1-2=-2 .
$$

On the other hand, if we assume $S$ is a subversion strategy with $|S| \geq 2$, then the remaining graph is empty. Therefore it contradicts to the definition of neighbor rupture degree.

From (21) and (22) we have $\operatorname{Nr}\left(P_{n}^{c}\right)=-1$.

The following theorem's proof is very similar to that of Theorem 14.

Theorem 15. Let $W_{1, n}$ be a wheel graph of order $n+1$. Then

$$
\operatorname{Nr}\left(W_{1, n}^{c}\right)=-1
$$

Theorem 16. Let $K_{m, n}$ be a complete bipartite graph. Then

$$
\operatorname{Nr}\left(K_{m, n}^{c}\right)= \begin{cases}2-n, & 2>n-m, \\ -m, & \text { otherwise. }\end{cases}
$$

Proof. It is obvious that $K_{m, n}^{c}=K_{m} \cup K_{n}$.

According to Corollary 7 we get the result.

Corollary 17. Let $K_{1, n}$ be a star graph of order $n+1$. Then

$$
\operatorname{Nr}\left(K_{1, n}^{c}\right)=-1
$$


Definition 18 (see [7]). The cartesian product $G=G_{1} \times G_{2}$ has $V(G)=V\left(G_{1}\right) \times V\left(G_{2}\right)$, and two vertices $\left(u_{1}, u_{2}\right)$ and $\left(v_{1}, v_{2}\right)$ of $G$ are adjacent if and only if either

$$
u_{1}=v_{1}, \quad u_{2} v_{2} \in E\left(G_{2}\right)
$$

or

$$
u_{2}=v_{2}, \quad u_{1} v_{1} \in E\left(G_{1}\right) .
$$

Theorem 19. Let $P_{2} \times P_{3 a}$ be a cartesian product with $a \in Z^{+}$. Then

$$
\operatorname{Nr}\left(P_{2} \times P_{3 a}\right)=a-1 .
$$

Proof. Let $S$ be a subversion strategy of $P_{2} \times P_{3 a}$ and $|S|=r$. There are two cases according to the number of elements in S.

Case 1. Let $1 \leq r \leq a$. Then $N[S] \leq 4 r$ and $w \leq 2 r$.

Since $c\left(\left(P_{2} \times P_{3 a}\right) / S\right) \geq(|V(S)|-|N(S)|) / w \geq((2 .(3 a)-$ $4 r) / 2 r)=3 a / r-2$ and

$$
\begin{aligned}
& w\left(\frac{P_{2} \times P_{3 a}}{S}\right)-|S|-c\left(\frac{P_{2} \times P_{3 a}}{S}\right) \\
& \quad \leq 2 r-r-\left(\frac{3 a}{r}-2\right) \\
& \quad=r+2-\frac{3 a}{r} .
\end{aligned}
$$

Let $f(r)=r+2-3 a / r$. $f$ is an increasing function since $f^{\prime}(r)=1+3 a / r^{2}>0$. So it takes its maximum value at $r=a$. Then $f(a)=a-3 a / a+2=a-1$. Hence,

$$
\operatorname{Nr}\left(P_{2} \times P_{3 a}\right) \leq a-1 .
$$

Case 2. Let $a \leq r \leq\left|V\left(P_{2} \times P_{3 a}\right)\right|$. Then $c\left(\left(P_{2} \times P_{3 a}\right) / S\right) \geq 1$ and $w\left(\left(P_{2} \times P_{3 a}\right) / S\right) \leq 2(3 a)-4 a-(r-a)=3 a-r$, thus we obtain $w\left(\left(P_{2} \times P_{3 a}\right) / S\right)-|S|-c\left(\left(P_{2} \times P_{3 a}\right) / S\right) \leq 3 a-r-r-1=3 a-2 r-1$.

Let $f(r)=3 a-2 r-1$. Since $f^{\prime}<0, f$ is a decreasing function, so it takes its maximum value at $r=a$. Then $f(a)=$ $3 a-2 a-1=a-1$

$$
\operatorname{Nr}\left(P_{2} \times P_{3 a}\right) \leq a-1
$$

From (30) and (31) we have

$$
\operatorname{Nr}\left(P_{2} \times P_{3 a}\right) \leq a-1 .
$$

It is obvious that there exist $S^{*}$ such that $\left|S^{*}\right|=a, w\left(\left(P_{2} \times\right.\right.$ $\left.\left.P_{3 a}\right) / S^{*}\right)=2 a$ and $c\left(\left(P_{2} \times P_{3 a}\right) / S^{*}\right)=1$ so

$$
\operatorname{Nr}\left(P_{2} \times P_{3 a}\right) \geq a-1 .
$$

From (32) and (33) we have $\operatorname{Nr}\left(P_{2} \times P_{3 a}\right)=a-1$.

The following theorems' proofs are very similar to that of Theorem 19.
Theorem 20. Let $P_{2} \times P_{3 a+1}$ be a cartesian product with $a \in Z^{+}$. Then

$$
\operatorname{Nr}\left(P_{2} \times P_{3 a+1}\right)=a-2 .
$$

Theorem 21. Let $P_{2} \times P_{3 a+2}$ be a cartesian product with a $\epsilon$ $Z^{+}$. Then

$$
\operatorname{Nr}\left(P_{2} \times P_{3 a+2}\right)=a-1 .
$$

Theorem 22. Let $K_{m}$ and $K_{n}$ be two complete graphs with $(m \leq n)$. Then

$$
\operatorname{Nr}\left(K_{m} \times K_{n}\right)=1-n
$$

Proof. Let $S$ be a subversion strategy of $K_{m} \times K_{n}$ and let $|S|=r$. We have two cases according to the cardinality of $S$.

Case 1. If $0 \leq r<m-1$, then $w\left(\left(K_{m} \times K_{n}\right) / S\right)=1$ and $c\left(\left(K_{m} \times K_{n}\right) / S\right) \geq(m-r)(n-r)$, so we have

$$
\begin{aligned}
& w\left(\frac{K_{m} \times K_{n}}{S}\right)-|S|-c\left(\frac{K_{m} \times K_{n}}{S}\right) \\
& \quad \leq 1-r-(m-r)(n-r) .
\end{aligned}
$$

Let $f(r)=1-r-m n+(m+n) r-r^{2}$. Since $f(r)$ is an increasing function in $(0, m-1)$, it takes its maximum value at $r=m-1$ and $f(m-1)=1-n$. Thus we get

$$
\operatorname{Nr}\left(K_{m} \times K_{n}\right) \leq 1-n
$$

Case 2. If $m-1 \leq r \leq(m-1)(n-1)$, then $w\left(\left(K_{m} \times K_{n}\right) / S\right)=1$ and $c\left(\left(K_{m} \times K_{n}\right) / S\right) \geq n-m+1$. So we have $w\left(\left(K_{m} \times K_{n}\right) / S\right)-$ $|S|-c\left(\left(K_{m} \times K_{n}\right) / S\right) \leq 1-r-(n-m+1)=m-n-r$.

Let $f(r)=m-n-r$. Since $f(r)$ is a decreasing function, it takes its maximum value at $r=m-1$ and $f(m-1)=1-n$. Thus we get

$$
\operatorname{Nr}\left(K_{m} \times K_{n}\right) \leq 1-n
$$

From (38) and (39) we have

$$
\mathrm{Nr} \leq 1-n
$$

There exist $S^{*}$ such that $r=m-1, w\left(\left(K_{m} \times K_{n}\right) / S^{*}\right)=1$ and $c\left(\left(K_{m} \times K_{n}\right) / S^{*}\right)=n-m+1$, thus we have

$$
\mathrm{Nr} \geq 1-n
$$

From (40) and (41) we get $\mathrm{Nr}=1-n$.

Definition 23 (see [9]). The tensor product $G_{1} \otimes G_{2}$ of two simple graphs $G_{1}$ and $G_{2}$ is the graph with $V\left(G_{1} \otimes G_{2}\right)=V_{1} \times$ $V_{2}$ and where in $\left(u_{1}, u_{2}\right)$ and $\left(v_{1}, v_{2}\right)$ are adjacent in $G_{1} \otimes G_{2}$ if, and only if, $u_{1}$ is adjacent to $v_{1}$ in $G_{1}$ and $u_{2}$ is adjacent to $v_{2}$ in $G_{2}$.

Theorem 24. Let $P_{3} \otimes P_{n}$ be a tensor product of $P_{3}$ and $P_{n}$ and $n \equiv 0(\bmod 4)$. Then

$$
\operatorname{Nr}\left(P_{3} \otimes P_{n}\right)=n-1 \text {. }
$$


Proof. Let $S$ be a subversion strategy of $P_{3} \otimes P_{n}$ and $|S|=r$ be the number of removing vertices from $P_{3} \otimes P_{n}$. There are two cases according to the number of elements in $S$.

Case 1. If $0 \leq r \leq(n / 2)$, then $w\left(\left(P_{3} \otimes P_{n}\right) / S\right) \leq n+r$ and $c\left(\left(P_{3} \otimes P_{n}\right) / S\right) \geq 1$. Thus we have

$$
w\left(\frac{P_{3} \otimes P_{n}}{S}\right)-|S|-c\left(\frac{P_{3} \otimes P_{n}}{S}\right) \leq n-1
$$

Case 2. If $(n / 2) \leq r \leq 3 n$, then $w\left(\left(P_{3} \otimes P_{n}\right) / S\right) \leq n+(n / 2)-$ $\left((r-(n / 2))=2 n-r\right.$ and $c\left(\left(P_{3} \otimes P_{n}\right) / S\right) \geq 1$. Thus we have

$$
w\left(\frac{P_{3} \otimes P_{n}}{S}\right)-|S|-c\left(\frac{P_{3} \otimes P_{n}}{S}\right) \leq 2 n-2 r-1 .
$$

Let $f(r)=2 n-2 r-1$ since $f^{\prime}(r)<0$ the function $f(r)$ is a decreasing function so it takes its maximum value at

$$
r=\left(\frac{n}{2}\right), \quad f\left(\frac{n}{2}\right)=n-1 .
$$

From (43) and (45) we get

$$
\operatorname{Nr}\left(P_{3} \otimes P_{n}\right) \leq n-1 .
$$

There exist $S^{*}$ such that $\left|S^{*}\right|=(n / 2), w\left(\left(P_{3} \otimes P_{n}\right) / S\right)=n+(n / 2)$ and $c\left(\left(P_{3} \otimes P_{n}\right) / S\right) \geq 1$ thus we have

$$
\operatorname{Nr}\left(P_{3} \otimes P_{n}\right) \geq n-1 \text {. }
$$

From (46) and (47) we get $\operatorname{Nr}\left(P_{3} \otimes P_{n}\right)=n-1$.

The following theorem's proof is very similar to that of Theorem 24.

Theorem 25. Let $P_{3} \otimes P_{n}$ be a tensor product of $P_{3}$ and $P_{n}$ and $n \neq 0(\bmod 4)$. Then

$$
\operatorname{Nr}\left(P_{3} \otimes P_{n}\right)=2 n-2\left\lceil\frac{(n+1)}{2}\right\rceil-1 .
$$

Theorem 26. Let the tensor product of $K_{m}$ and $K_{n}$ is $K_{m} \otimes K_{n}$. Then

$$
\operatorname{Nr}\left(K_{m} \otimes K_{n}\right)=\operatorname{Nr}\left(K_{m-1, n-1}\right)-1=\max \{m-5, n-5\} .
$$

Proof. Let $(a, b)$ be any vertex of $K_{m} \otimes K_{n}$ such that $a \in K_{m}$ and $b \in K_{n}$. The only vertices that are not adjacent to $(a, b)$ in $\left(K_{m} \otimes K_{n}\right)$ are $\left(a, c_{j}\right)$ with $(j=1,2, \ldots, n)$ and $\left(d_{i}, b\right)$ with $(i=1,2, \ldots, m)$, where $c_{j} \in V\left(K_{n}\right)$ and $d_{i} \in V\left(K_{m}\right)$.

The vertices $\left(a, c_{j}\right)$ are not adjacent to each other, neither do the vertices $\left(d_{i}, b\right)$. But these are adjacent to each other, so

$$
\begin{gathered}
\frac{\left(K_{m} \otimes K_{n}\right)}{\{a, b\}} \cong K_{m-1, n-1}, \\
\operatorname{Nr}\left(K_{m} \otimes K_{n}\right)=\operatorname{Nr}\left(K_{m-1, n-1}\right)-1 \\
=\max \{m-4, n-4\}-1 \\
=\max \{m-5, n-5\} .
\end{gathered}
$$

Definition 27 (see [10]). The composition of simple graphs $G$ and $H$ is the simple graph $G[H]$ with vertex set $V(G) \times V(H)$, in which $(u, v)$ is adjacent $\left(u^{\prime}, v^{\prime}\right)$ if and only if either $u u^{\prime} \in$ $E(G)$ or $u=u^{\prime}$ and $v v^{\prime} \in E(H)$.

Theorem 28. Let $P_{3}\left[P_{n}\right]$ be the composition of $P_{3}$ and $P_{n}$ with $n \geq 5$. Then neighbor rupture degree of $P_{3}\left[P_{n}\right]$ is

$$
\operatorname{Nr}\left(P_{3}\left[P_{n}\right]\right)= \begin{cases}1, & n \equiv 1(\bmod 4), \\ 0, & n \equiv 0,2,3(\bmod 4) .\end{cases}
$$

Proof. Let the vertex set of $P_{3}\left[P_{n}\right]$ be labeled as $P_{n}^{i}, P_{n}^{i i}$, and $P_{n}^{i i i}$.

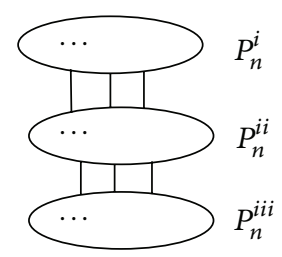

It can be easily seen that $P_{3}\left[P_{n}\right]$ is the sequential join of three disjoint path graphs, $P_{3}\left[P_{n}\right] \cong P_{n}+P_{n}+P_{n}$. Then, according to the Theorem 10 we get

$$
\begin{aligned}
\operatorname{Nr}\left(P_{3}\left[P_{n}\right]\right) & =\operatorname{Nr}\left(P_{n}+P_{n}+P_{n}\right) \\
& =\max \left\{\operatorname{Nr}\left(P_{n} \cup P_{n}\right), \operatorname{Nr}\left(P_{n}\right)\right\} .
\end{aligned}
$$

By Theorem 2, we have

$$
\operatorname{Nr}\left(P_{n}\right)= \begin{cases}0, & n \equiv 1(\bmod 4), \\ -1, & n \equiv 0,2,3(\bmod 4) .\end{cases}
$$

To conclude the proof we need to find $\operatorname{Nr}\left(P_{n} \cup P_{n}\right)$. Let $S$ be a subversion strategy of $P_{n} \cup P_{n}$. Since $P_{n} \cup P_{n}$ has two identical disjoint path graphs, let $x$ denote the number of removing vertices of each $P_{n}$ and let $|S|=2 x$.

Case 1. If $x \leq\lfloor(n-1) / 4\rfloor, w\left(\left(P_{n} \cup P_{n}\right) \backslash S\right) \leq 2 x+2$ and $c\left(\left(P_{n} \cup P_{n}\right) \backslash S\right) \geq\lceil(2 n-6 x) /(2 x+2)\rceil=\lceil(n-3 x) /(x+1)\rceil$, then we get

$$
\begin{aligned}
& w\left(\left(P_{n} \cup P_{n}\right) \backslash S\right)-|S|-c\left(\left(P_{n} \cup P_{n}\right) \backslash S\right) \\
& \quad \leq 2-\left\lceil\frac{n-3 x}{x+1}\right\rceil .
\end{aligned}
$$

Let $f(x)=2-(n-3 x) /(x+1)$, since $f(x)$ is an increasing function it takes its maximum value at $x=\lfloor(n-1) / 4\rfloor . f(\lfloor(n-$ $1) / 4\rfloor)=5-(n+3) /(\lfloor(n-1) / 4\rfloor+1)$. Since neighbor rupture degree has to be an integer, we have $\operatorname{Nr}\left(P_{n} \cup P_{n}\right) \leq\lceil 5-(n+$ $3) /(\lfloor(n-1) / 4\rfloor+1)\rceil$ and we get

$$
\operatorname{Nr}\left(P_{n} \cup P_{n}\right) \leq\left\{\begin{array}{l}
1, \quad n \equiv 1(\bmod 4), \\
0, n \equiv 0,2,3(\bmod 4) .
\end{array}\right.
$$

Case 2. If $x \geq\lfloor(n-1) / 4\rfloor, w\left(\left(P_{n} \cup P_{n}\right) \backslash S\right) \leq 2 x$, and $c\left(\left(P_{n} \cup\right.\right.$ $\left.\left.P_{n}\right) \backslash S\right) \geq 1$, thus we get

$$
\mathrm{Nr}\left(P_{n} \cup P_{n}\right) \leq-1
$$


From (56) and (57) we have

$$
\operatorname{Nr}\left(P_{n} \cup P_{n}\right) \leq \begin{cases}1, & n \equiv 1(\bmod 4), \\ 0, & n \equiv 0,2,3(\bmod 4) .\end{cases}
$$

There exist $S^{*}$ such that $\left|S^{*}\right|=2\lfloor(n-1) / 4\rfloor, w\left(\left(P_{n} \cup P_{n}\right) \backslash S\right)=$ $(2\lfloor(n-1) / 4\rfloor+1)$ and $c\left(\left(P_{n} \cup P_{n}\right) \backslash S\right)=2$ for $n \equiv 0,2,3(\bmod 4)$, $c\left(\left(P_{n} \cup P_{n}\right) \backslash S\right)=1$ for $n \equiv 1(\bmod 4)$.

Thus we have

$$
\operatorname{Nr}\left(P_{n} \cup P_{n}\right) \geq \begin{cases}1, & n \equiv 1(\bmod 4), \\ 0, & n \equiv 0,2,3(\bmod 4) .\end{cases}
$$

From (58) and (59) we get

$$
\operatorname{Nr}\left(P_{n} \cup P_{n}\right)= \begin{cases}1, & n \equiv 1(\bmod 4), \\ 0, & n \equiv 0,2,3(\bmod 4) .\end{cases}
$$

By (54) and (60) we conclude the proof.

Theorem 29. Neighbor rupture degree of composition of $K_{m}$ and any graph $G$ is

$$
\operatorname{Nr}\left(K^{m}[G]\right)=\operatorname{Nr}(G) .
$$

Proof. Let the vertex set of $K_{m}[G]$ be labeled as, $G^{i}, G^{i i}, \ldots, G^{m}$,
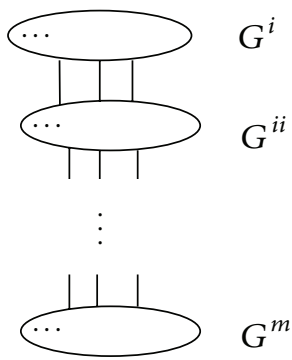

Let $S$ be a subversion strategy of $K_{m}[G]$. We have two cases according to the elements of $S$.

Case 1. Let we choose one element from any vertex set $G^{i}(i=$ $1,2, \ldots, m)$; if $u \in V\left(G^{i}\right)$ and $S=\{u\}$, then it removes all of other vertex sets. So it depends on only $G^{i}$ which we choose one element. Then we have

$$
\operatorname{Nr}\left(K_{m}[G]\right)=\operatorname{Nr}\left(G^{i}\right)=\operatorname{Nr}(G) .
$$

Case 2. Let we choose two elements from any vertex set $G^{i}(i=1,2, \ldots, m)$ and $G^{j}(j=1,2, \ldots, m)$ with $i \neq j$. Then $\left(K_{m}[G]\right) / S$ is empty set. It contradicts to the definition of neighbor rupture degree. Thus we obtain

$$
\operatorname{Nr}\left(K_{m}[G]\right)=\operatorname{Nr}(G) .
$$

Definition 30 (see [11]). An $n$th power of a graph $G$ is formed by adding an edge between all pairs of vertices of $G$ with distance at most $n$. If $n=2$ then it is called a second power of a graph also called a square.
Theorem 31. Neighbor rupture degree of $P_{n}^{2}(n>6)$ is

$$
\operatorname{Nr}\left(P_{n}^{2}\right)= \begin{cases}0, & n \equiv 1(\bmod 6) \\ -1, & \text { otherwise. }\end{cases}
$$

Proof. Let $S$ be a subversion strategy of $P_{n}^{2}$ and let $|S|=r$. There are two cases according to the number of elements of $S$.

Case 1. If $0 \leq r \leq\lceil n / 6\rceil-1$, then $w\left(P_{n}^{2} / S\right) \leq r+1, c\left(P_{n}^{2} / S\right) \geq$ $(n-5 r) /(r+1)$; then we get

$$
\begin{aligned}
& w\left(\frac{P_{n}^{2}}{S}\right)-|S|-c\left(P_{n}^{2} \backslash S\right) \\
& \quad \leq r+1-r-\frac{n-5 r}{r+1}=6-\frac{n+5}{r+1} .
\end{aligned}
$$

Let

$$
f(r)=6-\frac{n+5}{r+1}= \begin{cases}0, & n \equiv 1(\bmod 6), \\ <0, & \text { otherwise. }\end{cases}
$$

Case 2. If $=\lceil n / 6\rceil$, then $w\left(P_{n}^{2} / S\right) \leq r, c\left(P_{n}^{2} / S\right) \geq 1$; then we get

$$
\begin{aligned}
& w\left(\frac{P_{n}^{2}}{S}\right)-|S|-c\left(\frac{P_{n}^{2}}{S}\right) \\
& \quad \leq r-r-1 \\
& \quad=-1 \text { therefore } \mathrm{Nr} \leq-1 .
\end{aligned}
$$

Case 3. If $\lceil n / 6\rceil+1 \leq r \leq n$, then $w\left(P_{n}^{2} / S\right) \leq r-1, c\left(P_{n}^{2} / S\right) \geq 1$; then we get

$$
\begin{aligned}
& w\left(\frac{P_{n}^{2}}{S}\right)-|S|-c\left(P_{n}^{2} \backslash S\right) \\
& \quad \leq r-1-r-1 \\
& \quad=-2 \text { therefore } \mathrm{Nr} \leq-2 .
\end{aligned}
$$

According to (67), (68), and (69) we have

$$
\operatorname{Nr}\left(P_{n}^{2}\right) \leq \begin{cases}0, & n \equiv 1(\bmod 6) \\ -1, & \text { otherwise }\end{cases}
$$

There exist $S^{*}$ such that $\left|S^{*}\right|=\lceil(n-1) / 6\rceil, w\left(P_{n}^{2} / S^{*}\right)=\lceil n / 6\rceil$, $c\left(P_{n}^{2} / S^{*}\right)=1 ;$ then

$$
w\left(\frac{P_{n}^{2}}{S^{*}}\right)-\left|S^{*}\right|-c\left(\frac{P_{n}^{2}}{S^{*}}\right)= \begin{cases}0, & n \equiv 1(\bmod 6), \\ 1, & \text { otherwise. }\end{cases}
$$

By (70) and (71) we get the result,

$$
\begin{aligned}
\operatorname{Nr}\left(\frac{P_{n}^{2}}{S}\right) & =\max \left\{w\left(\frac{P_{n}^{2}}{S}\right)-|S|-c\left(\frac{P_{n}^{2}}{S}\right)\right\} \\
& = \begin{cases}0, & n \equiv 1(\bmod 6), \\
1, & \text { otherwise. }\end{cases}
\end{aligned}
$$




\section{Conclusion}

In this study, we investigate the neighbor rupture degree of graphs obtained by graph operations. The graph operations are used to obtain new graphs. Union, join, complement, composition, power, cartesian product, and tensor product are taken into consideration in this work. These operations are performed to various graphs and their neighbor rupture degrees were determined.

\section{References}

[1] F. Harary, Graph Theory, Addison-Wesley, New York, NY, USA, 1969.

[2] C. A. Barefoot and H. R. Entringer, "Vulnerability in graphs: a comparative survey," Journal of Combinatorial Mathematics and Combinatorial Computing, pp. 25-33, 1987.

[3] M. B. Cozzens and S.-S. Y. Wu, "Vertex-neighbor-integrity of powers of cycles," Ars Combinatoria, vol. 48, pp. 257-270, 1998.

[4] Y. Li, S. Zhang, and X. Li, "Rupture degree of graphs," International Journal of Computer Mathematics, vol. 82, no. 7, pp. 793803, 2005.

[5] G. Bacak-Turan and A. Kirlangic, "Neighbor rupture degree and the relations between other parameters," Ars Combinatoria, vol. 102, pp. 333-352, 2011.

[6] G. Bacak Turan, Vulnerability parameters in graphs [Ph.D. thesis], Ege University, 2010.

[7] G. Chartrand and L. Lesniak, Graphs and Digraphs, Chapman \& Hall, Orange, Calif, USA, 3rd edition, 1996.

[8] F. Buckley and F. Harary, Distance in Graphs, 1990.

[9] R. Balakrishan and K. Ranganathan, A Textbook of Graph Theory, 1999.

[10] J. A. Bondy and U. S. R. Murty, Graph Theory with Applications, Macmillan Press, London, UK, 1976.

[11] Handbook of Graph Theory, Discrete Mathematics and its Applications (Boca Raton), CRC Press, Boca Raton, Fla, USA, 2004. 


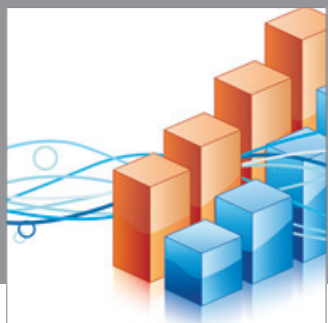

Advances in

Operations Research

mansans

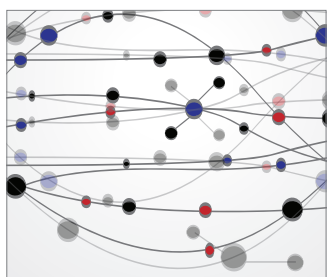

The Scientific World Journal
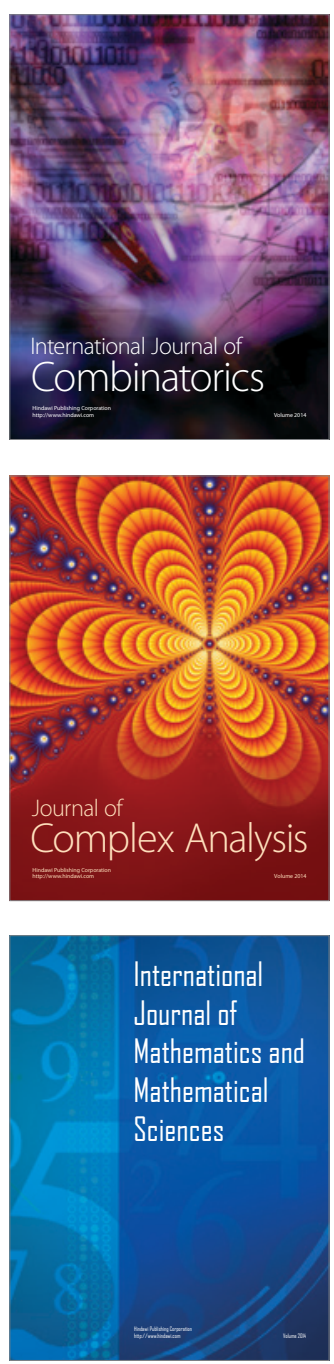
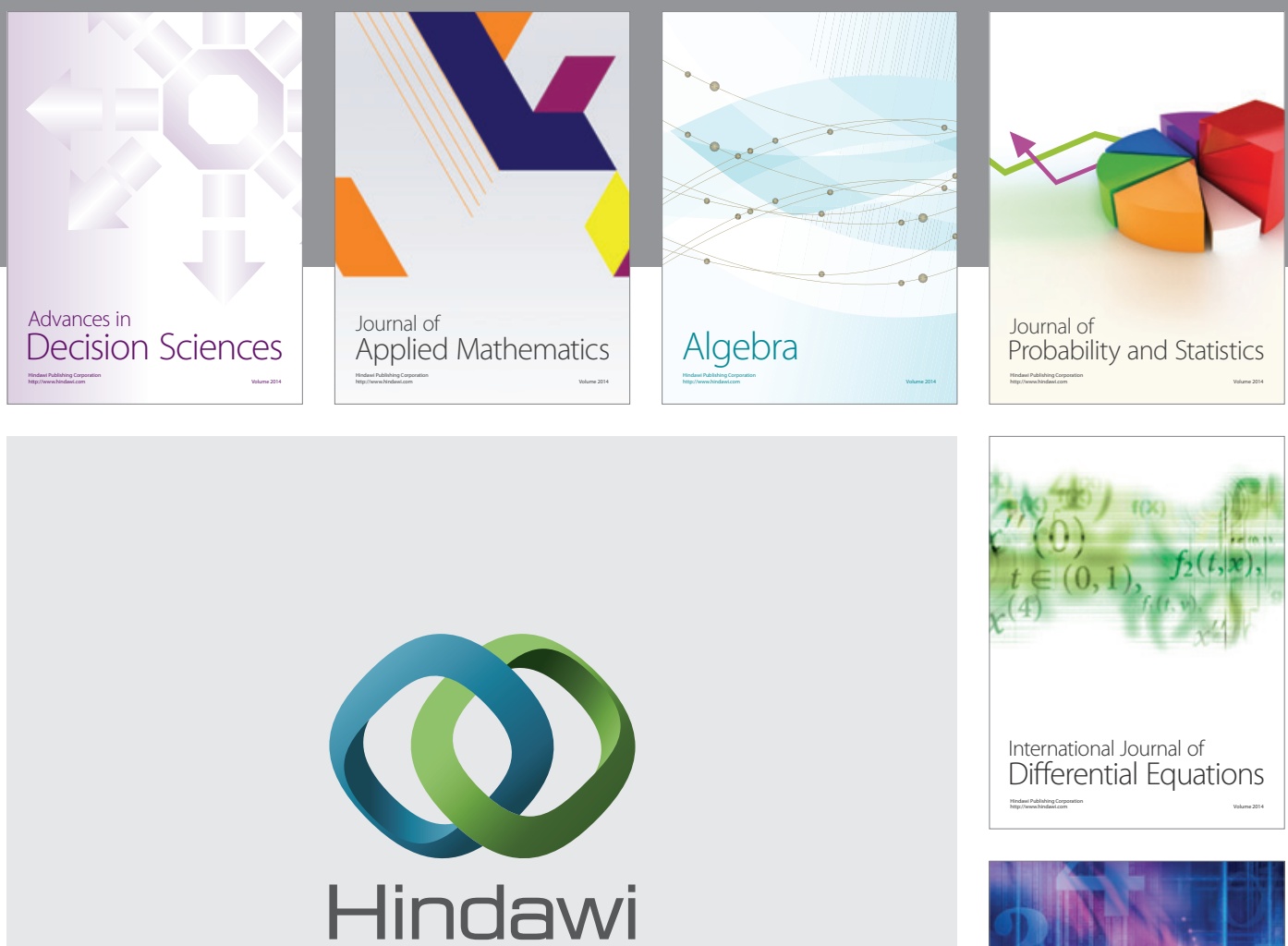

Submit your manuscripts at http://www.hindawi.com
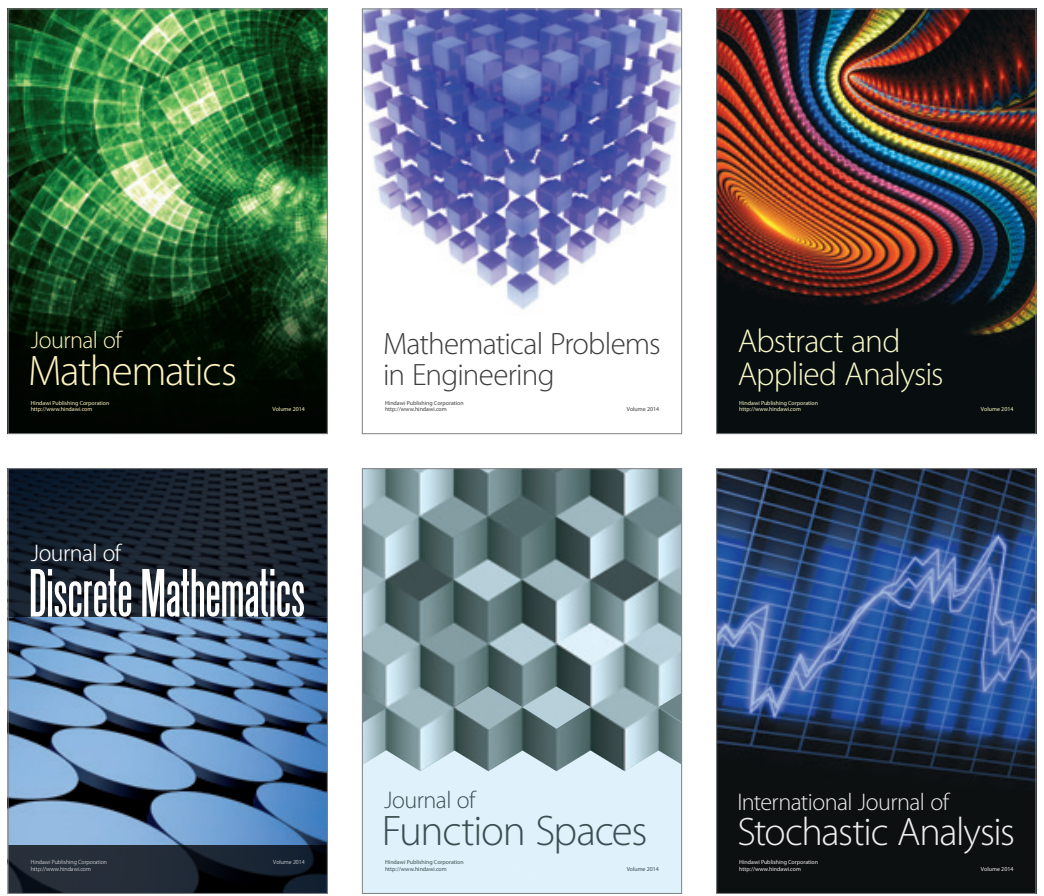

Journal of

Function Spaces

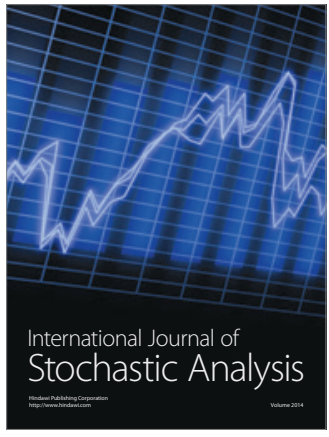

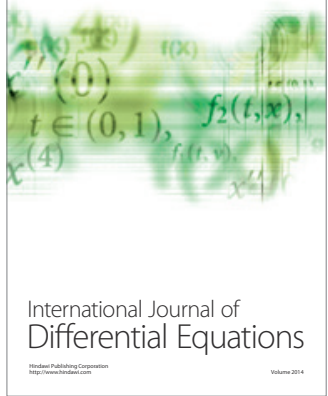
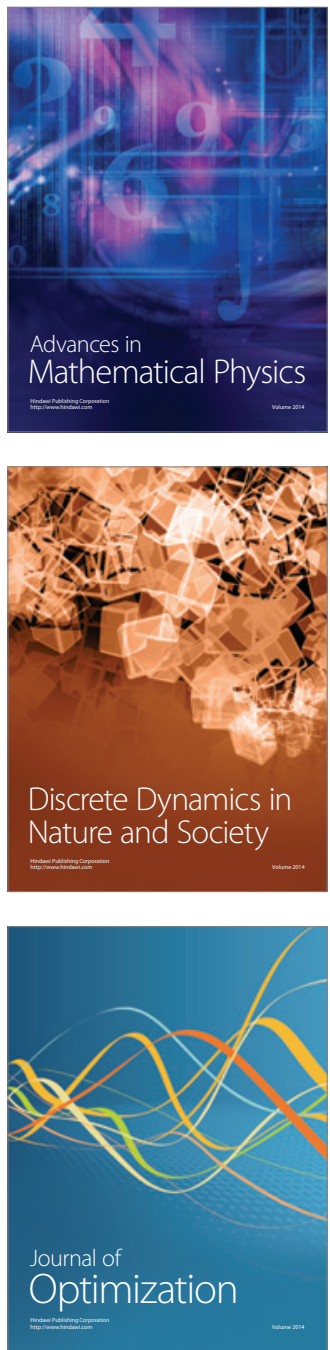